SOCIAL RESEARCH REPORTS

ISSN: 2066-6861 (print), ISSN: 2067-5941 (electronic)

PREVALENCE OF GROUP B STREPTOCOCCUS AND LEVEL OF RELIGIOSITY OF JEWISH MOTHERS IN NORTHERN ISRAEL - IS THERE A CONNECTION?

Adi SHARABI-NOV, Stefan COJOCARU

Social Research Reports, 2021, Vol. 13, Issue 1, pp. 9-17

The online version of this article can be found at:

www. researchreports.ro

https://doi.org/10.33788/srr13.1.1

Published by:

Expert Projects Publishing House

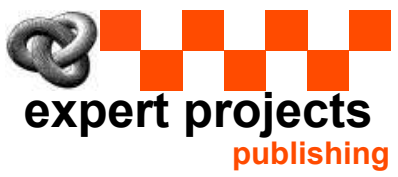

Covered by Index Copernicus International

www.indexcopernicus.com

Directory of Open Access Journals

www.doaj.org

On behalf of:

Center for Program and Social Development

Aditional services and information about Social Research Reports

can be found at:

www.researchreports.ro 


\title{
PREVALENCE OF GROUP B STREPTOCOCCUS AND LEVEL OF RELIGIOSITY OF JEWISH MOTHERS IN NORTHERN ISRAEL \\ - IS THERE A CONNECTION?
}

\author{
Adi SHARABI-NOV ${ }^{1}$, Stefan COJOCARU ${ }^{2}$
}

\section{Abstract}

Group B streptococcus (GBS) during pregnancy is associated with increased risk of preterm delivery and is a leading cause of invasive infections in newborns. Rates of vaginal GBS prevalence in pregnant women vary among ethnic groups and geographical regions, with a range of $0.5-38 \%$. The aim is to assess the prevalence rate of GBS bacteria among Jewish pregnant mothers in northern Israel, according to their level of religiosity. A cross-sectional study was pursued in 303 Jewish mothers at Ziv Medical Center, Safed. of whom 89 (29.4\%) were secular, $59(19.5 \%)$ traditional, 60 (19.8\%) Orthodox and 95 (31.3\%) ultra-Orthodox, from December 2019-July 2020. All participants were tested for GBS from pregnancy week 35 as part of the routine medical care at their Health Maintenance Organization (HMO) and submitted the GBS test result upon arrival at the hospital delivery room. The overall prevalence of GBS was 33.7\%: $16.9 \%$ of the secular mothers, $25.4 \%$ of the traditional women and $46.5 \%$ of the Orthodox and ultraOrthodox group $(\mathrm{p}<0.001)$. In a univariate regression model the risk of GBS carriage in Orthodox mothers was 2.5 times higher and 6 times higher in ultraOrthodox mothers than in secular mothers ( $p$ for trend $<0.001$ ). The prevalence of GBS was directly and significantly associated with the level of the birthing mother's religiosity. This study shows the need for systematic GBS screening of all pregnant women, particularly of Orthodox and ultra-Orthodox women, prior to their arrival in the delivery room.

Keywords: Group B streptococcus, pregnant woman, level of religiosity.

\footnotetext{
${ }^{1}$ Ziv Medical Center, Safed, Israel and Tel-Hai Academic College, Upper Galilee, ISRAEL; Department of Sociology and Social Work, Alexandru Ioan Cuza University from Iasi, ROMANIA. E-mail: adi_nov@hotmail.com

${ }^{2}$ Department of Sociology and Social Work, Alexandru Ioan Cuza University from Iasi, ROMANIA. E-mail: contact@stefancojocaru.ro
} 


\section{Introduction}

Group B streptococcus (GBS) is found in vaginal flora and in the anus, and in healthy adults it is generally not associated with morbidity (Schuchat, 1999). Pregnant women infected by GBS may suffer from urinary tract infection, fever $\left(38^{\circ} \mathrm{c}\right.$ and above), preterm labor or prolonged rupture of the membranes (PROM) $\geq 18$ hours. They are also at a higher risk for preterm delivery (defined as delivery before 37 weeks of gestation age). Additionally, they may infect their newborn with GBS. All positive GBS-bearing women attending the delivery room should be treated with intrapartum antibiotics prophylactic (IAP). (ACOG, 2020; Phares et al., 2008; Schuchat, 1999).

\section{Literature review}

GBS is the major cause of neonatal morbidity and mortality, both in neonates aged 0-6 days (EOD - early-onset disease) and in neonates aged 7-90 days (LOD - late-onset disease). The majority $(90 \%)$ of EOD morbidity is observed within 24 hours of birth and is characterized by direct infection of the newborn by their mothers (Verani et al., 2010; Walker et al., 2019)prolonged rupture of membranes, chorioamnionitis and GBS colonization. Risk-based predictive models are used to identify and screen infants. Late onset neonatal sepsis (LONS. The major diseases in neonates associated with GBS exposure are sepsis (Basu, 2015; Walker et al., 2019)prolonged rupture of membranes, chorioamnionitis and GBS colonization. Risk-based predictive models are used to identify and screen infants. Late onset neonatal sepsis (LONS, meningitis (Berardi et al., 2007; Hernández et al., 2011) and pneumonia (Berardi et al., 2007).

GBS prevalence among pregnant women varies according to ethnic groups and geographical regions, with a range of $0.5-38 \%: 22.4 \%$ in Africa, $19.7 \%$ in the U.S., $16.7 \%$ in Eastern Mediterranean countries, $19.0 \%$ in Europe and $11.1 \%$ in Asia (Kwatra et al., 2016), with a maternal GBS colonization worldwide pooled prevalence of $18 \%$ (Russell et al., 2017)fetus, and newborn. This article, the second in a series estimating the burden of GBS, aims to determine the prevalence and serotype distribution of GBS colonizing pregnant women worldwide. Methods. We conducted systematic literature reviews (PubMed/Medline, Embase, Latin American and Caribbean Health Sciences Literature [LILACS], World Health Organization Library Information System [WHOLIS], and Scopus. For infants the worldwide incidence rate of GBS disease is 0.49/1000 live births, with EOD incidence at $0.41 / 1000$ live births and LOD at 0.26/1000 live births (Madrid et al., 2017)despite declines due to intrapartum antibiotic prophylaxis (IAP.

A review of studies conducted in Israel showed that the prevalence of GBS among Jewish pregnant women in Israel rose from $2.6 \%$ in 1983 to $28.4 \%$ in 2020 , a more than tenfold increase (Weintraub et al., 1983; Rottenstreich et al., 2020). For EOD neonates, the annual incidence rate was 0.1/1000 live births in 1983 (Weintraub et al., 1983) and increased to $0.51 / 1000$ live births in 2020 (Schindler et al., 2017). In Israel, according to the directive of the Ministry of Health, there 
is no universal screening of pregnant women for GBS carriage only according to risk-base approach (Berlovitz, 2005)

The aim of the current study was to assess the prevalence of GBS bacteria among Jewish pregnant women in northern Israel, according to the level of religiosity.

\section{Sample and Methods}

This is a cross-sectional study based on the presence of the GBS bacterium in pregnant Jewish women from northern Israel who delivered at Ziv Medical Center and who underwent a GBS test at the Health Maintenance Organization (HMO) from gestational week 34 , as recommended by the family doctor or the community gynecologist.

Jewish women were enrolled between December 24, 2019 and July 6, 2020 during their stay in the maternity ward. Maternal medical records and sociodemographic details including the level of religiosity were extracted from a selfreported questionnaire completed by the pregnant woman during her post-delivery stay in the hospital. The level of self-reported religiosity was divided into secular, traditional, Orthodox and ultra-Orthodox.

\section{Methodology}

\section{Study population}

Of the 303 mothers recruited during the study period, 89 (29.4\%) were secular, $59(19.5 \%)$ traditional, 60 (19.8\%) Orthodox and 95 (31.3\%) ultra-Orthodox. Maternal age of secular and traditional mothers was significantly higher compered to Orthodox and ultra-Orthodox mothers (31.4 and 29.0 years, respectively, $\mathrm{p}$ $<0.001)$. Most of the pregnant women were born in Israel $(87.2 \%$ of secular and traditional mothers and $93.6 \%$ of Orthodox and ultra-Orthodox mothers). The parity was significantly higher among Orthodox and ultra-Orthodox mothers compared to secular and traditional (3.2 and 4.0 vs. 1.8 and 1.9 , respectively, $\mathrm{p}<0.001)$.

\section{Statistical Analysis}

The categorical variables were presented by frequency and percentages, while the continuous variables were represented using mean and standard deviation. Differences in GBS frequency was calculated using the independent samples t-test. A univariate logistic regression model, Odds Ratio (OR) estimates and 95\% confidence intervals $(95 \% \mathrm{CI})$ were calculated to examine the association between GBS carriage and level of religiosity. The data were analyzed using the SPSS version 25 . A value of $p<0.05$ was considered statistically significant. 


\section{Ethical considerations}

The study was approved by the ethics committee of Ziv Medical Center (Approval \# 0086-15-ZIV). All study participants signed an informed consent form prior to their enrolment.

\section{Results}

\section{Main outcome}

The overall prevalence of GBS was $33.7 \%$ and varied among the level of maternal religiosity groups: $16.9 \%$ in the secular group, $25.4 \%$ in the traditional group, $33.3 \%$ in the Orthodox group and $54.7 \%$ in the ultra-Orthodox group $(\mathrm{p}<0.001)$.

The prevalence of GBS was 2.5 times (95\% CI: 1.1-5.3) higher $(\mathrm{p}<0.05)$ among the Orthodox compared to the secular group and 6 times higher in the ultraOrthodox group (95\% CI: 3.0-11.9) $(\mathrm{p}<0.001)$ (Table 1, model 1). No significant differences in the prevalence of GBS were found between the secular and the traditional groups, nor between the Orthodox and the ultra-Orthodox groups. (Table 1, model 2). The combined Orthodox and ultra-Orthodox group was 3.41 (95\% CI: 2.1-5.7) times more likely to be GBS carriers than the combined secular and traditional group $(\mathrm{p}<0.001)$ (Table 1 , model 2).

Table 1. Univariate logistic regression models for examining the correlation between the prevalence of GBS infection and the level of maternal religiosity

\begin{tabular}{|c|c|c|c|c|}
\hline Model & Level of religiosity & OR & $95 \% \mathrm{Cl}$ & $\mathrm{p}$ \\
\hline 1 & Secular & Reference & & \\
\hline & Traditional & 1.68 & -3.7750 .7 & 070.2 \\
\hline & Orthodox & 2.47 & $1.14-5.34$ & 0.022 \\
\hline & Ultra-Orthodox & 5.97 & $3.00-11.85$ & $<0.001$ \\
\hline & P for trend & & & $<0.001$ \\
\hline 2 & Secular/Traditional & Reference & & \\
\hline & Orthodox/ultra-Orthodox & 3.41 & $2.05-5.68$ & $<0.001$ \\
\hline
\end{tabular}

Note: OR - odds ratio, CI - confidence interval

\section{Discussion}

The pooled prevalence of GBS in the study sample was 33.7\%, ranging from $16.9 \%$ in the secular group to $54.7 \%$ in the ultra-Orthodox group ( $\mathrm{p}$ for trend $<0.001$ ).

Combining the secular with traditional pregnant women the prevalence found in this study (20.3\%) is almost identical to the value Sefty et al (2016) determined in the Israeli national survey (23\%). However, GBS prevalence among Orthodox and ultra-Orthodox was significantly higher at $46.5 \%$, which is in the same range 
value reported in low and middle income countries such Brazil (51\%), South Africa (37\%) and Turkey (32\%) (Vieira et al., 2019routine screening for this bacterium in prenatal care before the onset of labor is recommended. However, many women present in labor without having undergone such testing during antenatal care, and the turnaround time of detection methods is insufficient for results to be obtained before delivery. Methods: Vaginal and anorectal specimens were collected from 270 pregnant women. Each sample was tested by Xpert GBS, qPCR, and culture for GBS detection. Results: The overall prevalence of maternal GBS colonization was $30.7 \%$ according to Xpert GBS, $51.1 \%$ according to qPCR, and $14.3 \%$ according to cultures. Considering the qPCR method as the reference, the Xpert GBS had a sensitivity of 53\% and specificity of $93 \%$. Positive Xpert GBS results were correlated to marital status (married or cohabitating; Mukesi et al., 2019; Kadanali et al., 2005)

While parity was higher in the Orthodox and ultra-Orthodox groups (and the two combined) compared to the secular and traditional groups (or the two combined), the difference in GBS rate between the two groups is quite high to be attributed only to the difference in parity. Additionally, the relationship between parity and GBS prevalence is controversial. Chen et al (2018) and Manzanares et al (2019) described a significant relationship between parity and GBS colonization, while in other studies this relationship was not been found to be significant (Jisuvei et al., 2020; Schindler et al., 2020; Namugongo et al., 2016).

Therefore, we suggest that various aspects of lifestyle may be responsible for our results. The aspects we hypothesized as being influential are: 1) Higher infection rates pre-pregnancy due to rituals marking the end of the menstrual period, 2) The potential impact of using ritual baths, and 3) Differences in nutrition.

Orthodox and ultra-Orthodox women use white fabric to swab the vaginal area following the end of their menstrual bleeding to verify that no residual bleeding has occurred. Due to the proximity of the vagina to the rectum, and the gastro-intestinal bearing of GBS, such ritual swabbing practice may cause vaginal cross-contamination. This ritual may thus be linked to higher prevalence of GBS pre-pregnancy among the Orthodox and ultra-Orthodox women that may be further carried on into their pregnancy. Vaginal cloth swabbing removes a significant amount of the natural vaginal bacterial flora, particularly the Lactobacillus that releases lactic acid and generates the protective acidic $\mathrm{pH}$ environment. Wiping out such shields against vaginal infection has already been found to allow for colonization by non-native vaginal bacteria including E-Coli and GBS (Neut et al., 2015). In fact, ritual vaginal swabbing was reported to be a practice in Kenyan and South African women that was found to be linked to a 2.5 times higher frequency of GBS (95\% CI: 0.99-6.24, p=0.053) (Cools \& Melin 2016). Van de Wijgert and colleague (2000) also found a $26.3 \%$ GBS frequency among women practicing such vaginal swabbing ritual in Zimbabwe compared to $14.7 \%$ among those who avoid such a ritual.

Immersion in ritual baths may also be a cause for increased GBS, as Orthodox and ultra-Orthodox men and women faithfully practice this ritual (Yosef, 2019). The hygienic conditions of ritual baths are questionable as the ritual occurs in warm water $\left( \pm 35^{\circ} \mathrm{c}\right)$, the water is seldomly replaced and the same water serves a large number of women. Given that GBS may leak from the gastro-intestinal tract of 
the bathing person and spread in the warm water, one can foresee the possibility of these facilities as a source of cross-contamination. Although pregnant women do not immerse in the ritual bath, they can be GBS carriers in two ways: prepregnancy or infected with GBS by their husbands who continue to immerse in the ritual bath. Since GBS was not monitored and checked in the ritual bath serving this study population, our assumption cannot be proven.

Orthodox and ultra-Orthodox people practice different nutritional habits compared to secular and traditional Jewish people, raising the possibility of a link to the difference in GBS rates between the former and latter groups, since GBS is a gastrointestinal bacterium. The major difference is the former groups' consumption of only "Kosher" food and the prohibition from consuming both meat and dairy foods at the same meal. To date, however, no research has been done on the relationship between "kosher" food and GBS. In addition, many food producers, suppliers, importers and restaurants in Israel are equipped with a "kosher" certificate (issued by law only by the Chief Rabbinate), (at: https://www. gov.il/he/departments/topics/national_kosher), primarily for commercial purposes in striving to reach as many consumers as possible. So, in fact, almost all the Jewish residents of Israel regularly consume Kosher food. There for "Kosher" food and the meat-dairy separation, could not be linked to the higher frequency of GBS prevalence among the Orthodox and ultra-Orthodox women.

\section{Conclusion}

A significantly higher prevalence of GBS was determined among Orthodox and ultra-Orthodox pregnant women arriving to the delivery room compared to their secular and traditional counterparts. These findings illuminate a clear correlation between level of religiosity and the susceptibility of GBS carriage during pregnancy. The origin of the higher prevalence appears to be related to strict adherence to the practice of certain religious rituals in the Jewish religion, but proving these relationships requires further evaluation. Yet, the study clearly proved the importance of systematic GBS screening at the HMOs for all pregnant women prior to their admission to the delivery room, particularly among the Orthodox and ultra-Orthodox Jewish women.

Due to vertical transmission from positive GBS carrier mother to newborn while passing through the birth canal or aspirating infected amniotic fluid during pregnancy (Vornhagen et al., 2017), the screening is also crucial to prevent newborn complications such as sepsis, meningitis and pneumonia.

\section{Acknowledgements}

The authors would like to thank Dr. Inbar Ben-Shahar, Dr. David Peleg and Suhila Ghanim from the department of Obstetrics and Gynecology at Ziv Medical Center in Safed for their help in conducting this research. 


\section{References}

American College of Obstetricians and Gynecologists. ACOG Committee Opinion: N. 797. (2020). Prevention of Group B Streptococcal Early-Onset Disease in Newborns. In Obstetrics and gynecology (Vol. 135, Issue 2). https://doi.org/10.1097/ AOG.0000000000003668

Basu, S. (2015). Neonatal sepsis: the gut connection. European Journal of Clinical Microbiology and Infectious Diseases, 34, 215-222. DOI: 10.1007/s10096-0142232-6.

Berardi, A., Lugli, L., Baronciani, D., Creti, R., Rossi, K., Ciccia, M., Gambini, L., Mariani, S., Papa, I., Serra, L., Tridapalli, E., \& Ferrari, F. (2007). Group B streptococcal infections in a northern region of Italy. Pediatrics, 120(3), e487-e493. DOI: 10.1542 /peds.2006-3246.

Berlovitz, I. (2005). Testing pregnant women for GBS. Israel Ministry of Health, guidelines No. 22/2005.

Chen, Z., Wu, C., Cao, X., Wen, G., Guo, D., Yao, Z., \& Ye, X. (2018). Risk factors for neonatal group $\mathrm{B}$ streptococcus vertical transmission: a prospective cohort study of 1815 mother-baby pairs. Journal of Perinatology, 38(10), 1309-1317. DOI: 10.1038/s41372-018-0182-z.

Cools, P., \& Melin, P. (2017). Group B Streptococcus and perinatal mortality. Research in Microbiology, 168(9-10), 793-801. DOI: 10.1016/j.resmic.2017.04.002.

Hernández, M. I., Sandoval, C. C., Tapia, J. L., Mesa, T., Escobar, R., Huete, I., Wei, X. C., \& Kirton, A. (2011). Stroke patterns in neonatal group B streptococcal meningitis. Pediatric Neurology, 44(4), 282-288. DOI: 10.1016/j.pediatrneurol.2010.11.002.

Jisuvei, S. C., Osoti, A., \& Njeri, M. A. (2020). Prevalence, antimicrobial susceptibility patterns, serotypes and risk factors for group B streptococcus rectovaginal isolates among pregnant women at Kenyatta National Hospital, Kenya; A cross-sectional study. BMC Infectious Diseases, 20(1), 1-10. DOI: 10.1186/s12879-020-05035-1.

Kadanali, A., Altoparlak, Ü., \& Kadanali, S. (2005). Maternal carriage and neonatal colonisation of group B streptococcus in eastern Turkey: Prevalence, risk factors and antimicrobial resistance. International Journal of Clinical Practice, 59(4), 437-440. DOI: 10.1111/j.1368-5031.2005.00395.x.

Kwatra, G., Cunnington, M. C., Merrall, E., Adrian, P. V., Ip, M., Klugman, K. P., Tam, W. H., \& Madhi, S. A. (2016). Prevalence of maternal colonisation with group B streptococcus: a systematic review and meta-analysis. The Lancet Infectious Diseases, 16(9), 1076-1084. DOI: 10.1016/S1473-3099(16)30055-X.

Madrid, L., Seale, A. C., Kohli-Lynch, M., Edmond, K. M., Lawn, J. E., Heath, P. T., Madhi, S. A., Baker, C. J., Bartlett, L., Cutland, C., Gravett, M. G., Ip, M., Le Doare, K., Rubens, C. E., Saha, S. K., Sobanjo-Ter Meulen, A., Vekemans, J., \& Schrag, S. (2017). Infant Group B Streptococcal Disease Incidence and Serotypes Worldwide: Systematic Review and Meta-analyses. Clinical Infectious Diseases, 65(Suppl 2), S160-S172. DOI: 10.1093/cid/cix656.

Manzanares, S., Zamorano, M., Naveiro-Fuentes, M., Pineda, A., Rodríguez-Granger, J., \& Puertas, A. (2019). Maternal obesity and the risk of group B streptococcal colonisation in pregnant women. Journal of Obstetrics and Gynaecology, 39(5), 628-632. DOI: 10.1080/01443615.2018.1552670. 
Mukesi, M., Iweriebor, B. C., Obi, L. C., Nwodo, U. U., Moyo, S. R., \& Okoh, A. I. (2019). Prevalence and capsular type distribution of Streptococcus agalactiae isolated from pregnant women in Namibia and South Africa. BMC Infectious Diseases, 19(1), 1-7. DOI: 10.1186/s12879-019-3809-6.

Namugongo, A., Bazira, J., Fajardot, Y., \& Joseph, N. (2016). Group B Streptococcus Colonization among Pregnant Women Attending Antenatal Care at Tertiary Hospital in Rural Southwestern Uganda. International Journal of Microbiology, 2016. DOI: $10.1155 / 2016 / 3816184$.

Neut, C., Verrière, F., Nelis, H. J., \& Coenye, T. (2015). Topical Treatment of Infectious Vaginitis: Effects of Antibiotic, Antifungal and Antiseptic Drugs on the Growth of Normal Vaginal Lactobacillus Strains. Open Journal of Obstetrics and Gynecology, 5, 173-180. DOI: 10.4236/ojog.2015.53024

Phares, C. R., Lynfield, R., Farley, M. M., Mohle-Boetani, J., Harrison, L. H., Petit, S., Craig, A. S., Schaffner, W., Zansky, S. M., Gershman, K., Stefonek, K. R., Albanese, B. A., Zell, E. R., Schuchat, A., \& Schrag, S. J. (2008). Epidemiology of invasive group B streptococcal disease in the United States, 1999-2005. JAMA Journal of the American Medical Association, 299(17), 2056-2065. DOI: 10.1001/ jama.299.17.2056.

Rottenstreich, M., Rotem, R., Srebnik, N., Farkash, R., Samueloff, A., \& GrisaruGranovsky, S. (2020). The recurrence risk of group B Streptococcus in consecutive deliveries. Journal of Maternal-Fetal and Neonatal Medicine, 33(13), 2263-2268. DOI: $10.1080 / 14767058.2018 .1548596$.

Russell, N.J., Seale, A.C., O’Driscoll, M., O’Sullivan, C., Bianchi-Jassir, F., GonzalezGuarin, J., Lawn, J. E., Baker, C. J., Bartlett, L., Cutland, C., Gravett, M. G., Heath, P. T., Le Doare, K., Madhi, S. A., Rubens, C. E., Schrag, S., Sobanjo-Ter Meulen, A., Vekemans, J., Saha, S. K., \& Ip, M. (2017). Maternal Colonization with Group B Streptococcus and Serotype Distribution Worldwide: Systematic Review and Meta-analyses. Clinical Infectious Diseases, 65, 100-111. DOI: 10.1093/cid/cix658

Schindler, Y., Rahav, G., Nissan, I., Madar-Shapiro, L., Abtibol, J., Ravid, M., \& Maor, Y. (2020). Group B Streptococcus serotypes associated with different clinical syndromes: Asymptomatic carriage in pregnant women, intrauterine fetal death, and early onset disease in the newborn. Plos One, 15(12), e0244450. DOI: 10.1371/ journal.pone.0244450.

Schuchat, A. (1999). Group B streptococcus. The Lancet, 353, 51-56

Sefty, H., Klivitsky, A., Bromberg, M., Dichtiar, R., Ami, M. Ben, Shohat, T., GlatmanFreedman, A., Anteby, E., Auslander, R., Ben Schachar, I., Borenstein, J., Chayen, B., Harel, L., Farah, N., Fishman, A., Golan, A., Gonen, O., Hagay, Z., Hakim, M., ... Ziv, N. (2016). Factors associated with choice of approach for Group B streptococcus screening. Israel Journal of Health Policy Research, 5(1), 1-9. DOI: 10.1186/s13584-016-0103-6.

van de Wijgert, J.H.H.M., Mason, P.R., Gwanzura, L., Mbizvo, M.T., Chirenje, Z.M., Iliff, V., Shiboski, S., \& Padian, N. S. (2000). Intravaginal Practices, Vaginal Flora Disturbances, and Acquisition. The Journal of Infectious Diseases, 181(2), 587594. DOI: $10.1086 / 315227$.

Verani, J. R., McGee, L., \& Schrag, S. J. (2010). Morbidity and Mortality Weekly Report Prevention of Perinatal Group B Streptococcal Disease. Morbidity and Mortality Weekly Report, 59(RR-10), 1-31. 
Vieira, L. L., Perez, A. V., Machado, M. M., Kayser, M. L., Vettori, D. V., Alegretti, A. P., Ferreira, C. F., Vettorazzi, J., \& Valério, E. G. (2019). Group B Streptococcus detection in pregnant women: Comparison of qPCR assay, culture, and the Xpert GBS rapid test. BMC Pregnancy and Childbirth, 19(1), 1-8. DOI: 10.1186/s12884019-2681-0.

Vornhagen, J., Adams Waldorf, K. M., \& Rajagopal, L. (2017). Perinatal Group B Streptococcal Infections: Virulence Factors, Immunity, and Prevention Strategies. Trends in Microbiology 25(11), 919-931. DOI: 10.1016/j.tim.2017.05.013.

Walker, O., Kenny, C. B., \& Goel, N. (2019). Neonatal sepsis. Paediatrics and Child Health (United Kingdom), 29(6), 263-268. DOI: 10.1016/j.paed.2019.03.003.

Weintraub, Z. Regev, R. Iancu, T.C. Ferne, M. Rabinowitz, B. S. (1983). Perinatal group B streptococcal infections in Israel. Isr J Med Sci, 19, 900-902.

Yosef, D. (2019). Purity: in halakhah and legend. https://www.shops.hidabroot.org/ items $/ 3369727-7 \% 94 \% \mathrm{D} 7 \% 98 \% \mathrm{D} 7 \% 94 \% \mathrm{D} 7 \% \mathrm{~A} 8 \% \mathrm{D} 7 \% 94 \% \mathrm{D} 7 \% 91 \% \mathrm{D} 7 \% 9$ 4\%D7\%9C\%D7\%9B\%D7\%94-D7\%95\%D7\%91\%D7\%90\%D7\%92\%D7\%93 $\% \mathrm{D} 7 \% 94$ 